\title{
Shadow Characteristics of a Column in an Infinite Length Room
}

\author{
Yoshihiko OHTANI*, Koh'ichi OHTA*, Akio TAKUBO** and Tetsuo YAMAYA* \\ * Department of Electrical Engineering, \\ College of Industrial Technology, Nihon University \\ 2-1, 1-Chome, Izumi-cho, Narashino-shi, Chiba 275 Japan \\ ** Matsushita Electronics Corporation \\ 1-1, Saiwai-cho, Takatsuki-shi, Osaka 569 Japan
}

This paper is based on the reports presented at the "1991 and 1992 Annual Conf. IEI Japan" and "1992 Tokyo-section Conf. IEI Japan".

\begin{abstract}
This paper deals with characteristics of the illuminance and the shadow factor within a shadow affected by reflected light, in an infinite length room which has a ribbon-shaped light source and a column (a shadow caster) of infinite length.

Calculations are done by Monte Carlo method which is widely used lately to predict the illuminance distribution of a room.

First, Monte Carlo method for numerical calculations is presented to obtain the illuminance within a shadow. Next, some experiments are made under the same conditions as the calculations. And, the experimental results are compared with the calculated results. As there is not a large difference seen between them, it is confirmed that the above method is suitable.

Finally, the characteristics of the illuminance and the shadow factor on the floor at the varied reflectances of the room surface and the shadow caster are obtained and examined.
\end{abstract}

\section{Introduction}

The shadow is one of the important factors in designing a lighting system to produce the best possible conditions for seeing things ${ }^{11}$.

One of the authors has tried by means of experiments and calculations to obtain direct illuminance values within the shadow produced on an illuminated plane when both light source and shadow caster have various geometrical shapes, and has examined the shadow characteristics ${ }^{2-8)}$.

Applying the shadow problem to the interior lighting design, however, requires that the shadow produced within a room is treated. That is, the reflected light by the ceiling, the wall and the floor has to be taken into consideration, as in a real room. Accordingly, the illuminance calculation within a shadow affected by the reflected light is done by Monte Carlo method which is widely used lately to predict the illuminance distribution of the room ${ }^{9)-12)}$.

These calculations of the illuminance within the shadow produced by an infinite length light source and an infinite length shadow caster set in an infinite length room are a step toward taking up a rectangular parallelepiped room in the actual scenes. The reasons why the infinite length room is chosen are that the illuminance values in this room are easily calculated by way of those of twodimensional surface ${ }^{13)}$ and that such a calculating method is considered as a useful method to obtain approximate values in the case of three-dimensional space.

First, Monte Carlo method for numerical calculations of the illuminance within a shadow is explained briefly.

Second, some experiments are made under the same conditions as the calculations. And, as there is not a large difference between the calculated results and the experimental results, it is confirmed that the above method is suitable ${ }^{14),}$ 15).

Finally, the characteristics of the illuminance and the shadow factor on the floor at the varied reflectances ${ }^{16), 17)}$ of the room surface and the shadow caster are obtained and examined. 


\section{Outline of calculation}

2.1 Setting up an infinite length room, a light source and a shadow caster
As shown in Fig. 1, the sizes of the infinite length room are 1.0 in width, 1.0 in height and infinite in length. And, the surfaces of the room are those of uniform diffuse reflection.

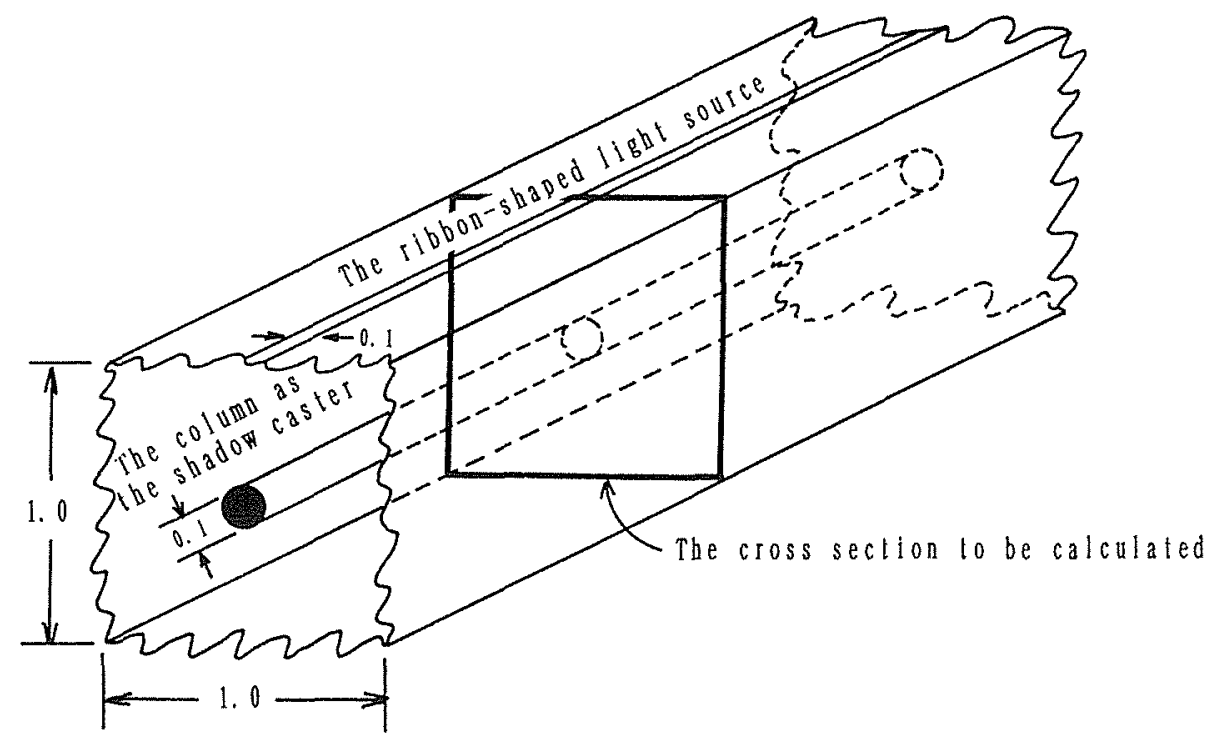

Fig. 1 Schematic of the infinite length room.

An infinite length diffuser ribbon-shaped light source with a width of 0.1 is installed in the center of the ceiling and its luminous intensity distribution is shown in Fig. 2 and is given by the following equation.

$$
I_{\theta}=I_{o}\left(a \cdot \cos \theta+b \cdot \cos ^{2} \theta\right)
$$

where; $I_{0}$ is the luminous intensity in the direction perpendicular to the source surface, per unit length of the source.

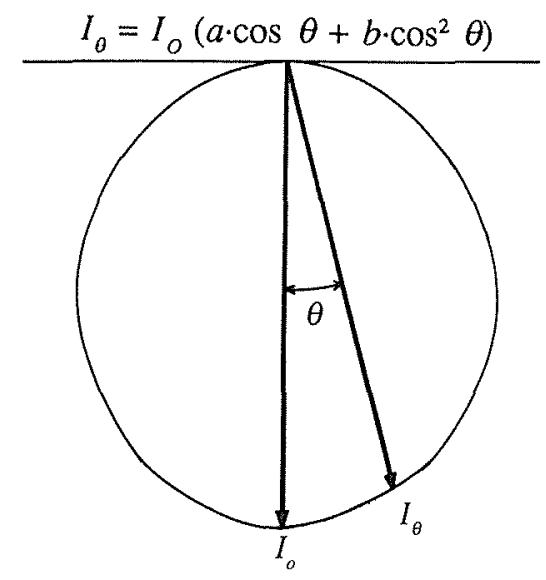

Fig. 2 The luminous intensity distribution curve of the infinite length diffuser ribbon-shaped light source.
$I_{\theta}$ is the luminous intensity in the direction $\theta$, per unit length of the source.

$$
a+b=1
$$

An infinite length column with a diameter of 0.1 is used as a shadow caster, which forms the shadow, and is set in the central part of the space in the room.

2.2 Illuminance in the infinite length room

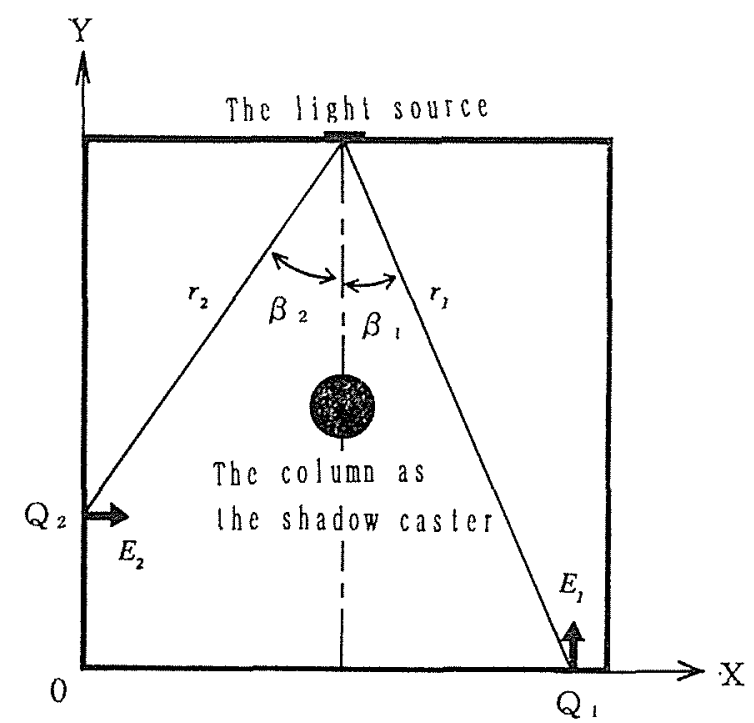

Fig. 3 The cross section of the infinite room. 
Fig. 3 shows a cross section of the infinite length room, where $r_{1}$ and $r_{2}$ are the distances from the light source to the illuminated points $Q$, and $Q_{2}, \beta$, and $\beta_{2}$ are the vertical angles in the direction of the illuminated points $Q_{l}$ and $Q_{2}, L$ is luminance of the light source and $w$ is the width of the light source, the direct illuminance $E$, at the point $Q_{1}$ on the floor and the direct illuminance $E_{2}$ at the point $Q_{2}$ on the wall are obtained as follows ${ }^{15}$.

$$
\begin{aligned}
& E_{1}=L \cdot w \cdot\left[\frac{a \pi}{2 r_{1}}+\frac{4 b}{3 r_{1}} \cdot \cos \beta_{1}\right] \cdot \cos ^{2} \beta_{1} \\
& E_{2}=L \cdot w \cdot\left[\frac{a \pi}{2 r_{2}}+\frac{4 b}{3 r_{2}} \cdot \cos \beta_{2}\right] \cdot \cos \beta_{2} \cdot \sin \beta_{2}
\end{aligned}
$$

In these equations, no variables related to the lengths of the infinite length room and the light source are found, so the illuminance values are constant despite the positions in the direction perpendicular to the cross section. Therefore, the calculation of the illuminance in the infinite length room is equivalently reduced to two dimensional space thought to be as one cross section alone.

As shown in Fig. 3, the locations of each surface, the light source and the shadow caster are indicated by a $X Y$ coordinate system whose origin is set up at the lower left-hand corner of the cross section.

\subsection{Illuminance calculation by Monte Carlo method}

In the illuminance calculation by Monte Carlo method, the flux emitted from a light source regards as a group of many particles. That is, the particles are defined by the photon bundle which transports a given quantity of energy per unit time ${ }^{13),}{ }^{18)}$. And, as for each particle, the direction of emission from the light source, the direction of emission after reflection on the surfaces such as the ceiling, the walls and the floor, and decision of reflection and absorption on the surfaces are decided by using the probability density function including the uniform random numbers.

Besides, the elements which have divided the surfaces of the room in equal parts are considered. The illuminance values at each position on the surfaces in the room are regarded as proportional to the number of the particles which collided with each element.

(1) Direction of the particles emitted from the light source

By the luminous intensity distribution of the equation (1), the number of the particles in the direction $\theta$ is

$$
P_{\theta}=P_{o}\left(a \cdot \cos \theta+b \cdot \cos ^{2} \theta\right)
$$

where; $P_{o}$ is the number of the particles in the direction perpendicular to the light source surface.

$P_{\theta}$ is the number of the particles in the direction $\theta$.

$a+b=1$
Because the above equation can be regarded as the probability density function, the equation related to the uniform random numbers $\xi$ is obtained as follows.

$$
\xi=\int_{-\frac{\pi}{2}}^{\theta} P_{o}\left(a \cdot \cos \theta+b \cdot \cos ^{2} \theta\right) d \theta
$$

Where $P_{0}$ is given to normalize the probability $P_{\theta}$ as

$$
\xi=\int_{-\frac{\pi}{2}}^{\frac{\pi}{2}} P_{\theta} d \theta=1
$$

Therefore, the relation of emitting direction $\theta$ to the uniform random numbers $\xi$ is determined as

$$
\xi=\frac{b \cdot \sin 2 \theta+4 a \cdot \sin \theta+4 a+b(2 \theta+\pi)}{8 a+2 b \pi}
$$

(2) Decision of reflection and absorption on the surface of the room

When the reflectance is $\rho$ and the uniform random number is $\xi, \xi \geqq \rho$ is considered to have been absorbed and $\xi<\rho$ reflected the particles.

(3) The direction of the particles emitted after reflecting

Since the surfaces of the room suppose to produce uniform diffuse reflection, the following equation is obtained in the same way as the equation (4).

$$
P_{\theta}=P_{0} \cos \theta
$$

So, the equation related to the uniform random numbers $\xi$ is obtained as follows.

$$
\xi=\int_{-\frac{\pi}{2}}^{\theta} P_{o} \cdot \cos \theta \cdot d \theta
$$

After fixing $P_{o}$ by the equation (6), the relation of emitting direction $\theta$ to the uniform random numbers $\xi$ is determined as

$$
\xi=(\sin \theta+1) / 2
$$

(4) Reflection and absorption of the particles on the surface of the shadow caster

When the straight line which indicates the flying locus of the particle emitted from the light source or the surface of the room intersects in the circle which indicates the cross section of the column as the shadow caster, it is considered that the particle collides with a certain point on the shadow caster. As for reflection and absorption, the same idea as the above items (2) and (3) is applicable supposing a tangential plane on the collided point of the shadow caster (See Fig. 4)

\section{(5) Optimization of the calculation}

In calculating, the total number of the particles emitted from the light source and the number of the elements obtained by dividing each surface of the room equally 


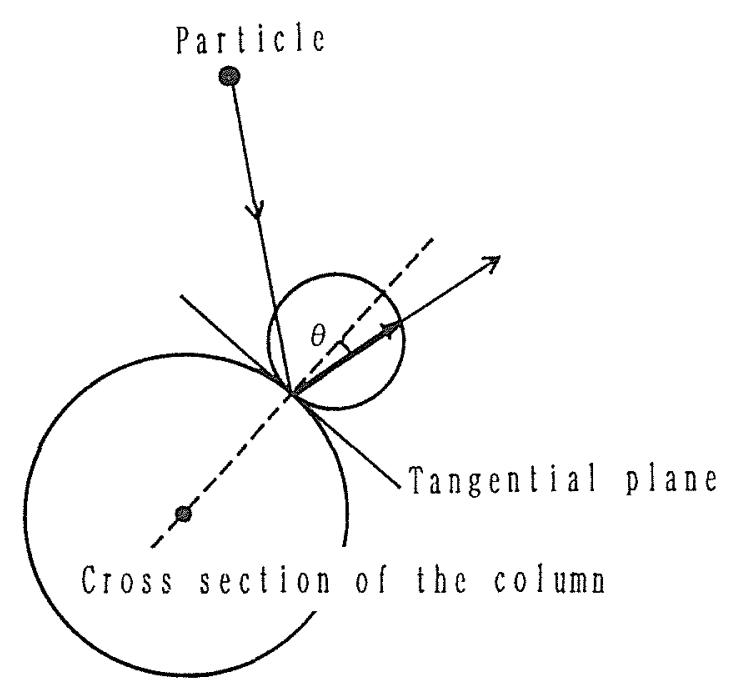

Fig. 4 Reflection of the particle on the column.

are related to the accuracy of the calculation and the CPU time. When the total number of the particles increases, the accuracy improves, but it takes longer CPU time. When the number of the elements is small, the detailed variations of the illuminance are not recognized, and when the number of the elements is large, the accuracy drops because of the decrease of the number of the particles per element.

Considering the accuracy of the calculation and the CPU time synthetically proves that five hundred thousand of particles and forty elements per surface are optimum ${ }^{18)}$. Under these conditions, CPU time used for the illuminance calculation in the cases of all surfaces with $0 \%$ reflectance and no shadow caster is 2 hours and 46 minutes (PC-9801RX by the use of BASIC compiler) and the differences from the theoretical values obtained by contour integration method ${ }^{19)}$ in the same cases are less than $3 \%$.

\section{Comparison between the calculated values and the experimental values}

Experiments are made under nearly the same conditions as those of calculations in order to see whether or not the above calculating methods are correct. And, the experimental results are compared with the calculated results ${ }^{14)}$.

\subsection{Outline of the experiments}

For the experiments, the model room with a width of $0.4 \mathrm{~m}$, a height of $0.4 \mathrm{~m}$ and a length of $5.8 \mathrm{~m}$ is used for the infinite length room. On the ceiling, walls and floor, well diffused Japanese paper with a reflectance of about $80 \%$ is pasted for simulation. And neither end of the room has a board.
A diffused ribbon-shaped light source with a size of $0.04 \mathrm{~m} \times 5.8 \mathrm{~m}$ is installed in the center of the ceiling. The emitting part of the light source is made of milk white acrylic board of $5 \mathrm{~mm}$ thickness and with a transmittance of about $30 \%$, and three $110 \mathrm{~W}$ fluorescent lamps are installed in the lamp box located on the opposite side of the emitting surface. The average luminance on the emitting surface is about $950 \mathrm{~cd} / \mathrm{m}^{2}$ and the uniformity ratio on it is about 0.92 . And, it is found by the measurement that the luminous intensity distribution of the light source is expressed approximately by the equation (1) with $a=0.72$ and $b=0.28$.

A column with a diameter of $0.05 \mathrm{~m}$ and a length of $5.8 \mathrm{~m}$ is used as the shadow caster which forms the shadow, and is set in the central part of the room space and also in parallel with the light source. Two kinds of shadow casters are used; the one is a mat painted black with a reflectance of about $0 \%$ and the other pasted with Japanese paper of a big diffusion rate with a reflectance of about $80 \%$.

For an illuminance meter, an integrated optical detector of $10 \mathrm{~mm}$ in diameter (Type 529-2-5 by Bell \& Howell) is used. The measurements of the illuminance are made at 20 points $0.02 \mathrm{~m}$ apart on the floor of the cross section located in the center of the model room.

3.2 Comparison between the calculated values and the experimental values

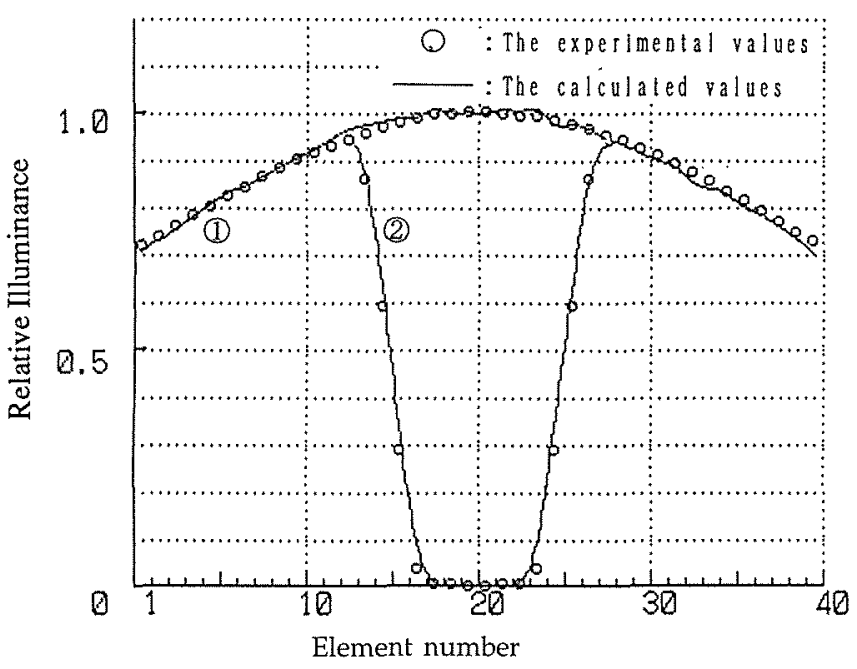

(1): Without the shadow caster

(2): With the shadow caster of the reflectance $0 \%$

Fig. 5 The illuminance distribution curves when the reflectance of the room is $0 \%$.

For example, Fig. 5 and Fig. 6 show the relative illuminance distribution curves on the floor. In these figures, the marks of $\bigcirc$ are the experimental values and are the calculated values. 


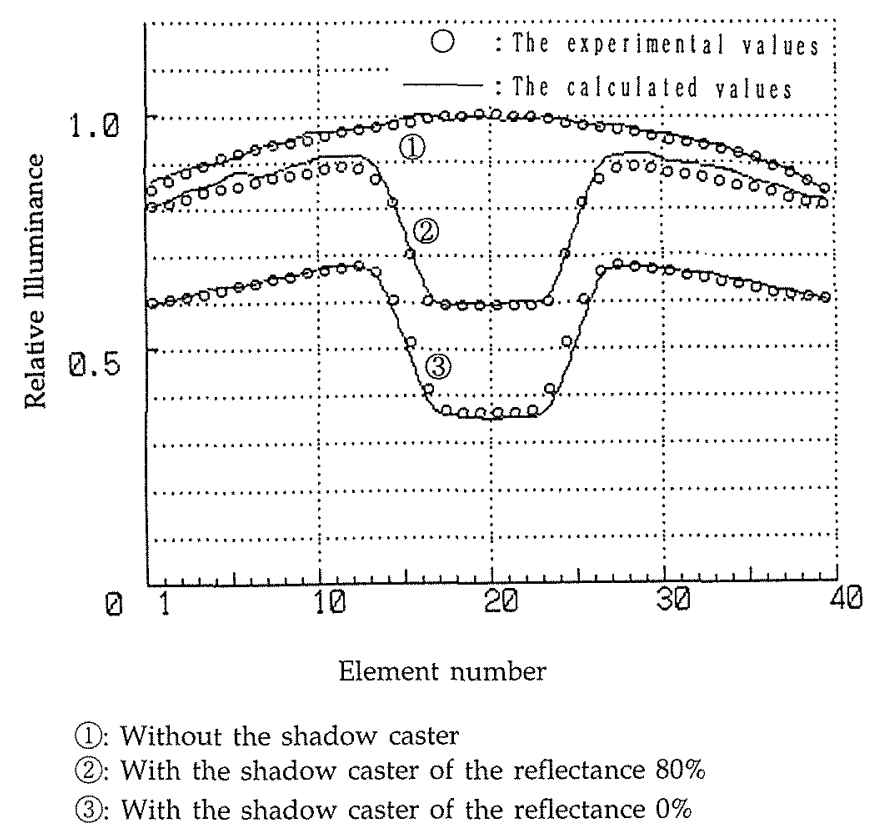

Fig. 6 The illuminance distribution curves when the reflectance of the room is $80 \%$.

Fig. 5 shows the case where only the direct illuminance existed without the room surfaces. This means that the reflectance of the surfaces in the room is $0 \%$ on calculation. Shown in Fig. 5(1) and (2) are the curves when the shadow caster is not set and when the shadow caster with a reflectance of about $0 \%$ is set respectively.

Fig. 6 shows the case when the reflectance of the surfaces in the room is $80 \%$. The curves (1), (2) and (3) shown in Fig. 6 are those when the shadow caster is not set, when the shadow caster with a reflectance of about $80 \%$ is set and when the shadow caster with a reflectance of about $0 \%$ is set respectively.

It is found that the experimental values agree closely with the calculated ones in both Fig. 5 and Fig. 6. As examples, the error rate curves of the calculated values to the experimental values are shown in Fig. 7 (a), (b) and (c) which indicate those on the curves (1), (2) and (3) in Fig. 6 respectively. The maximum error rate of (a) is $3.1 \%$ and the average $1.2 \%$, and similarly, (b) is $6.0 \%$ and $2.8 \%$ and (c) is $10.8 \%$ and $2.5 \%$. Though the maximum error rate in Fig. 7 (c) is over $10 \%$, the absolute error at the same point is as low as 0.056 , so it can be neglected. Therefore, it is confirmed that the illuminance values of calculations are correct.

\section{Shadow characteristics in the infinite length room}

As mentioned above, it is confirmed that the illuminance calculation method in the infinite length room by

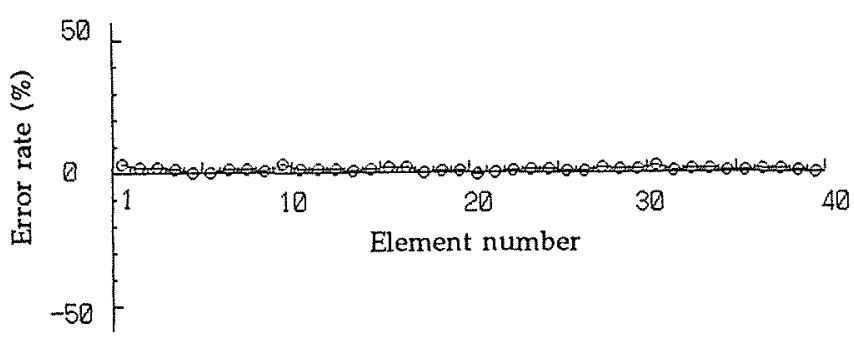

(a) : Without the shadow caster

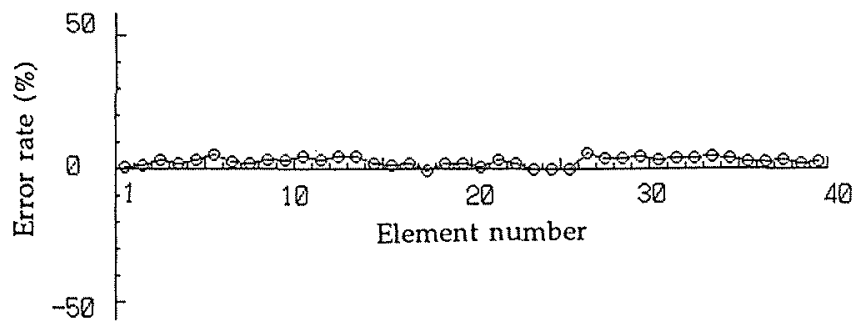

(b) : With the shadow caster of the reflectance $80 \%$

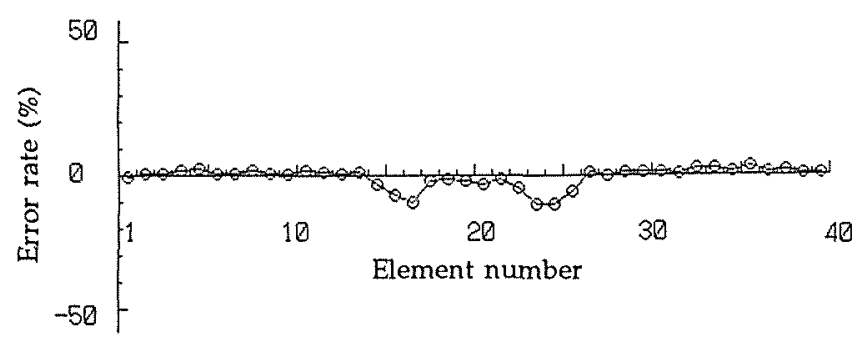

(c): With the shadow caster of the reflectance $0 \%$

Fig. 7 The error rate curves of the calculated values to the experimental values when the reflectance of the room is $80 \%$

Monte Carlo method is suitable. So, the illuminance values on the floor are calculated at the varied reflectances of the room surfaces and the shadow caster, and the shadow characteristics are obtained by these values and are examined.

\subsection{Shadow factor}

The shadow factor quantitatively indicates the state of the shadow and is also defined by the ratio of the illuminance reduced by the shadow to the unshaded illuminance ${ }^{201,21}$. That is, the shadow factor $S$ is given by the following equation. 


$$
S=\left(E_{o}-E_{S}\right) / E_{o}
$$

where: $E_{o}$ is the unshaded illuminance and $E_{S}$ is the residue illuminance within the shadow.

Thus, the value of the shadow factor becomes 1.0 within the umbra, between 1.0 and 0.0 within the penumbra and 0.0 in the ultraumbra.

In this paper, the illuminance distribution curves and the shadow factor distribution curves are used for an examination.

4.2 The case of varied reflectances of the surfaces in the room

Here, the following conditions are set.

(1) The infinite length room, the light source and the shadow caster are the same sizes as those described in the paragraph 2.1 .

(2) The luminous intensity distribution of the light source is the same as that described in the paragraph 3.1.

(3) The reflectance of the shadow caster is fixed by $0 \%$.

(4) The reflectances of the surfaces in the room are variable.

In Fig. 8, (a) shows the illuminance distribution curves on the floor when the shadow caster is not set, (b) shows the illuminance distribution curves on it when the shadow caster is set, and (c) shows the shadow factor distribution curves on it, where the reflectances of the surfaces in the room are taken as the parameter. The illuminance distribution curves of (a) and (b) are shown by the relative value with the maximum value of 1.0 when the shadow caster is not set and when the reflectances of the surfaces in the room are all $80 \%$.

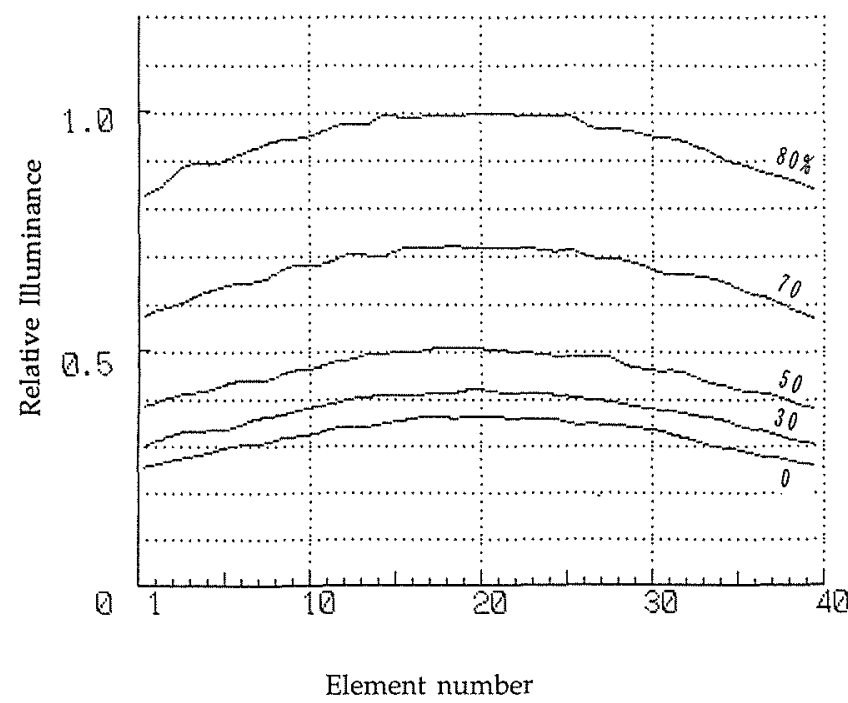

(a) : The illuminance distribution curves without the shadow caster

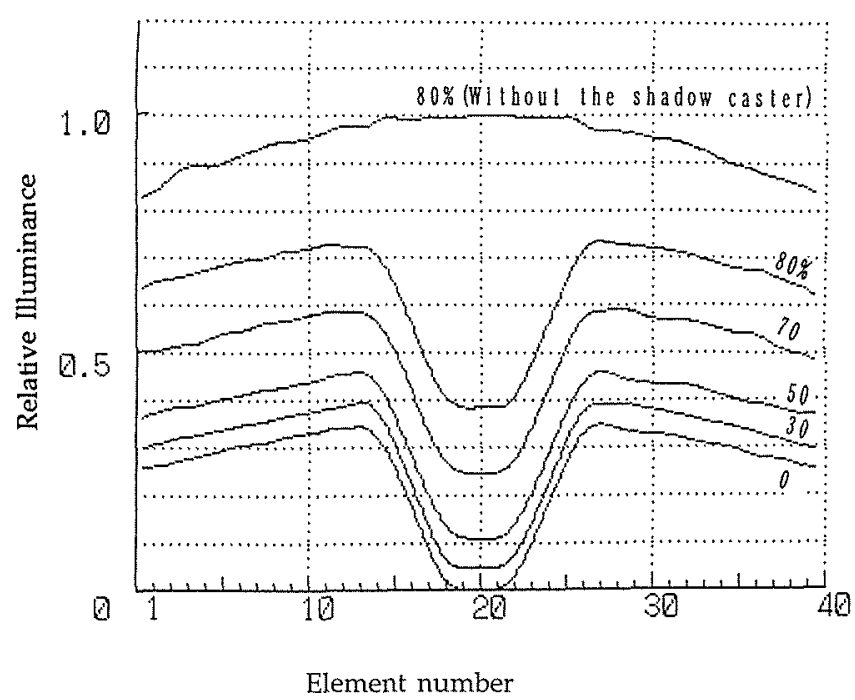

(b) : The illuminance distribution curves with the shadow caster of the reflectance $0 \%$

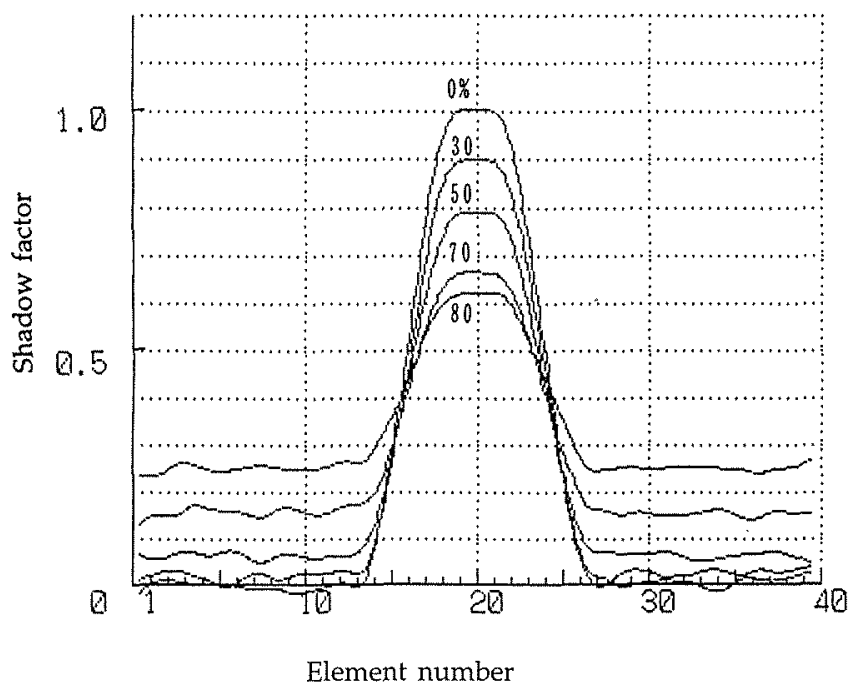

(c) : The shadow factor distribution curves

Fig. 8 The illuminance distribution curves and the shadow factor distribution curves when the reflectances of the room vary.

In the line graphs of Fig. 9, (a) shows the illuminance variations when the shadow caster is not set, (b) shows the illuminance variations when the shadow caster is set, and (c) shows the variations of the shadow factor, which correspond to the reflectances of the surfaces in the room. In these cases, the 1st element (the closest point to the origin) and the 20th element (the center point) on the floor are taken as the parameters.

The illuminance characteristics in the case of "no 


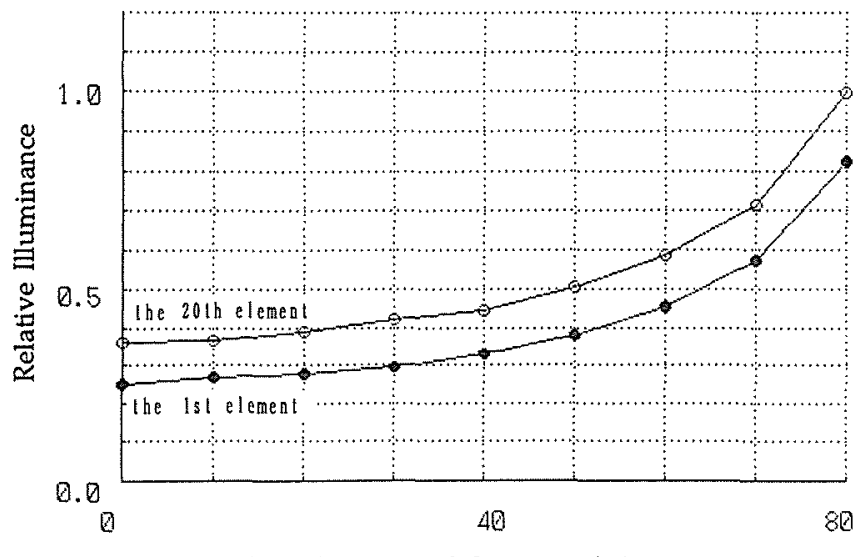

The reflectance of the room (\%)

(a): The variations of the illuminance without the shadow caster

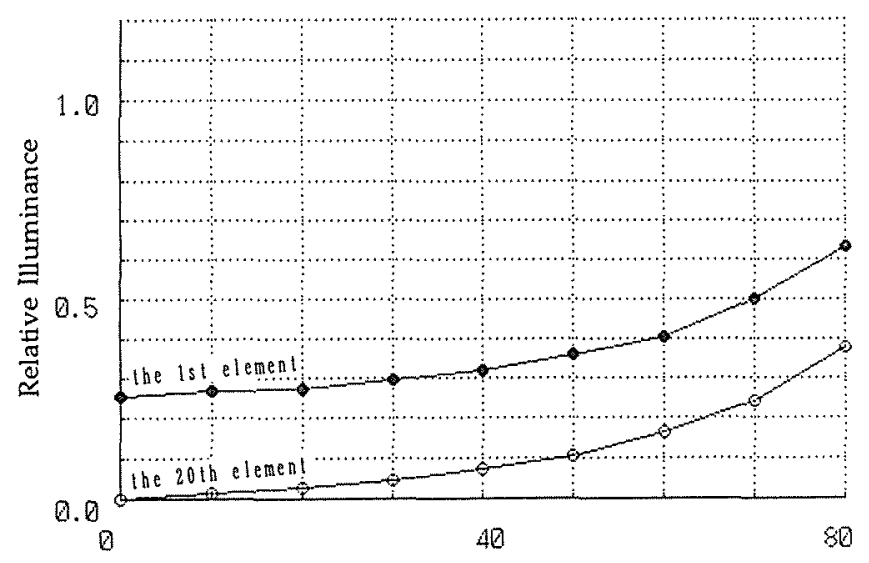

The reflectance of the room (\%)

(b) : The variations of the illuminance with the shadow caster of the reflectance $0 \%$

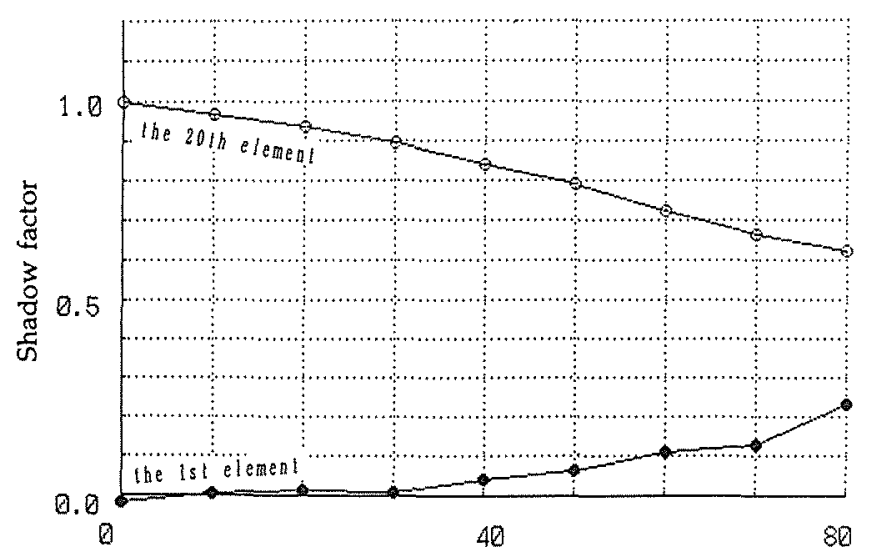

The reflectance of the room (\%)

(c) : The variations of the shadow factor

Fig. 9 The variations of the illuminance and the shadow factor corresponded to the reflectance of the room. shadow" shown in Fig. 8 (a) and Fig. 9 (a) tell that the higher the reflectance, the larger the illuminance value. The increase of the illuminance caused by changing the reflectance of the room from $0 \%$, namely, the case where only the direct light exists, to $80 \%$ is about 0.6 both at the 1 st element and at the 20th element. So, the degree of the influence of the reflected light to the illuminance is found out.

From the comparison between Fig. 8 (a) and Fig. 8 (b) or between Fig. 9 (a) and Fig. 9 (b), it is evident that a shadow is created at the central part just under the shadow caster, in any reflectance of the room, because the shadow caster shuts out the direct light. In the other part, only a drop in the illuminance caused by reduction of the reflected light is found, since the direct light is not shut at all. The increase of the illuminance which was brought about by the change of the reflectance of the room from $0 \%$ to $80 \%$ is about 0.4 both at the 1 st element and at the 20th element. In the case of the reflectance of $0 \%$, especially, the illuminance value at the central part is zero, that is, the umbra occurs, and no decrease of the illuminance value is observed at either end at all, that is, the shadow does not occur.

The shadow factor distribution curve in Fig. 8 (c) shows the effect of the reflectances over the shadow more clearly than comparing Fig. 8 (a) of the illuminance distribution curves without the shadow caster with Fig. 8 (b) of those with the shadow caster. In the case of the reflectance of $0 \%$, especially, it is obvious that the shadow factor at the central part is 1.0 , that is, the umbra has occurred, and the shadow factor at both ends is 0.0 , that is, shadow has not come out at all.

In Fig. 9 (c), when the reflectance of the room changes from $0 \%$ to $80 \%$, the shadow factor at the 20 th element decreases from 1.0 to about 0.6 and the shadow factor at the 1 st element increases from 0.0 to about 0.2 conversely, that is, the difference between the maximum value and the minimum value of the shadow factor on the floor becomes smaller by the effect of the reflectance on the room surfaces.

\subsection{The case of varied reflectances of the shadow caster}

The conditions set are as follows.

(1) The sizes of the infinite length room, the light source and the shadow caster are the same as those mentioned in the paragraph 2.1.

(2) The luminous intensity distribution of the light source is the same as that mentioned in the paragraph 3.1.

(3) The reflectances of the surfaces in the room are set at $70 \%$ at the ceiling, $50 \%$ at the walls and $30 \%$ at the floor.

(4) The reflectance of the shadow caster is variable.

In Fig. 10, (a) shows the illuminance distribution curves when the reflectance of the shadow caster varies and when the shadow caster is not set and (b) shows the shadow 


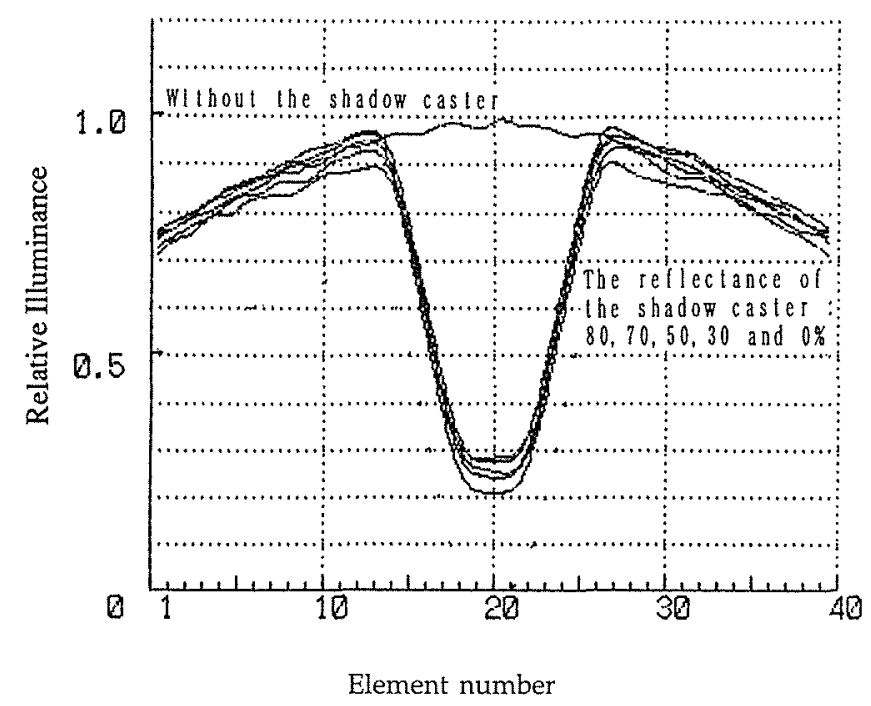

(a): The illuminance distribution curves with the shadow caster of varied reflectance and without the shadow caster

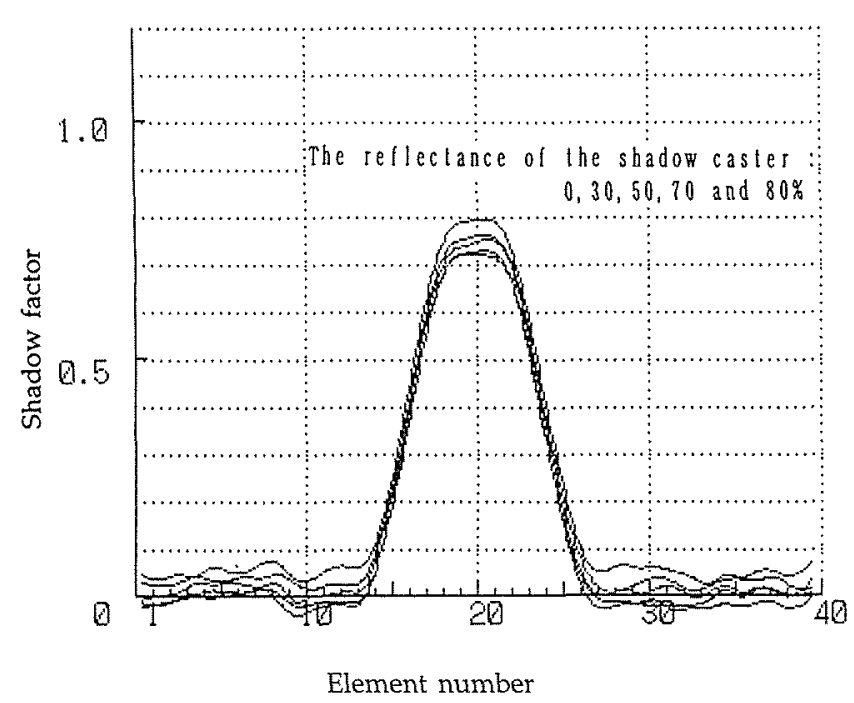

(b) : The shadow factor distribution curves

Fig. 10 The illuminance distribution curves and the shadow factor distribution curves when the reflectances of the room are set $70 \%$ at the ceiling, $50 \%$ at the walls and $30 \%$ at the floor.

factor distribution curves.

In the line graphs of. Fig. 11, (a) shows the illuminance variations and (b) shows the variations of the shadow factor, which correspond to the reflectance of the shadow caster each, in the case of the 1st element and 20th element on the floor being taken as the parameter.

The increase of the illuminance caused by the variations of the reflectance of the shadow caster from $0 \%$ to

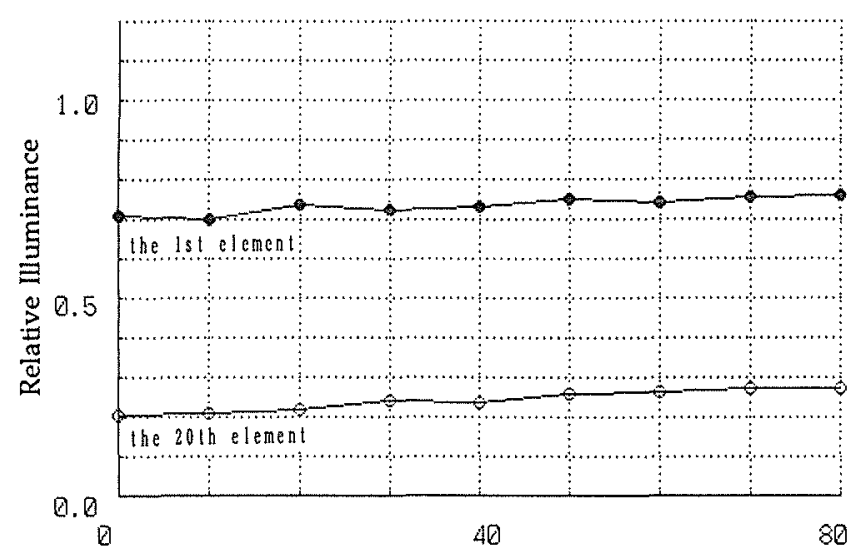

The reflectance of the shadow caster (\%)

(a): The variations of the illuminance

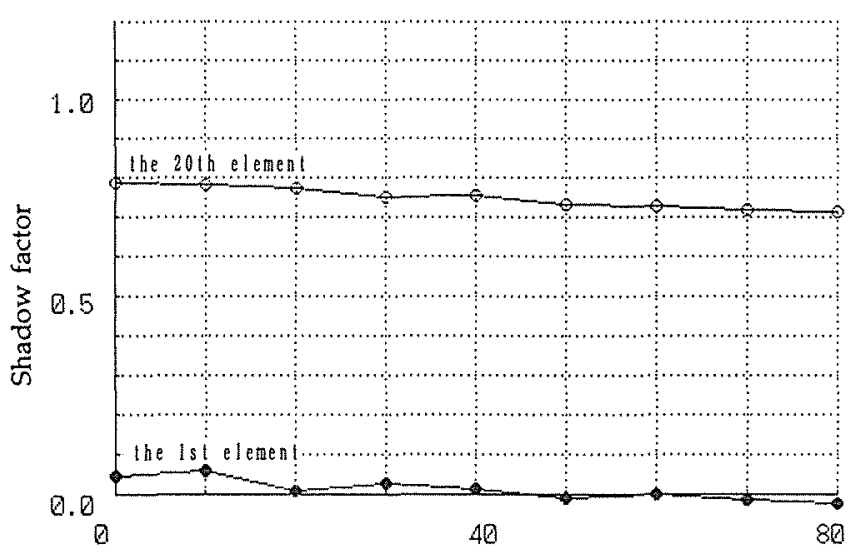

The reflectance of the shadow caster (\%)

(b): The variations of the shadow factor

Fig. 11 The variations of the illuminance and the shadow factor corresponded to the reflectance of the shadow caster.

$80 \%$ is about 0.1 both at the 1st element and at the 20th element, and the decrease of the shadow factor is about 0.15 at the 1 st element and is about 0.1 at the 20th element. So, the illuminance distribution and the shadow factor distribution on the floor are not affected so much by the reflectance variations of the shadow caster.

As shown in Fig. 10 (a), however, when the reflectance of the shadow caster becomes more than $70 \%$, the illuminance values are larger than those of the case without the shadow caster, at the elements between 1st and 7th, and between 33rd and 40th. This proves existence of the reflected light by the shadow caster. For that reason, the shadow factor takes the minus value, as shown in Fig. 10 (b) and Fig. 11 (b). 


\section{Conclusion}

The illuminance calculations within the shadow produced by the infinite length light source and the infinite length shadow caster set in the infinite length room are done, as an illuminance calculation within the shadow affected by reflected light. Comparing the calculated results with the experimental results under the same conditions makes it evident that the calculation method is proper.

Furthermore, as the examples of the calculated results, the distributions of the illuminance and the shadow factor on the floor at the varied reflectance of the room surfaces and the shadow caster are obtained and examined.

The increase of the illuminance caused by the change of the reflectances of the room from $0 \%$ to $80 \%$ is about 0.6 when the shadow caster is not set and is about 0.4 when the shadow caster is set. And, the difference between the maximum value and the minimum value of the shadow factor on the floor decreases from 1.0 to 0.4 .

It is found that the reflectance change of the shadow caster little affects the illuminance distribution and the shadow factor distribution on the floor of the room.

The authors's next task will be to obtain the shadow characteristics in the rectangular parallelepiped room of three dimensional space, which are conditions close to the actual scenes, and to compare them with those in the infinite length room.

\section{Acknowledgment}

The authors wish to thank the late Dr. Yoshiteru Fujiwara of Nihon University for the academic advices and encouragement he gave us.

\section{References}

(1) Moon, P. and Spencer, D.E.: "Lighting Design", Addison-Wesley Press (1948) 306 and 326

(2) Fujiwara, Y. and Ohtani, Y.: "Illuminance in the Shadow Formed by a Horizontal Disk (Using a Linear Light Source)", J. Illum. Engng. Inst. Japan 58-2 (1974) 49 57 (in Japanese)

(3) Fujiwara, Y. and Ohtani, Y.: "Characteristics of the Shadow Produced by a Horizontal Disk Setting under a Horizontal Linear Light Source", J. Illum. Engng. Inst. Japan 61-2 (1977) 92 98 (in Japanese)

(4) Fujiwara, Y. and Ohtani, Y.: "Some Characteristics of the Shadow Produced by a Horizontal Disk Setting under a Vertical Linear Light Source" ${ }^{\prime}$ J. Illum. Engng. Inst. Japan 61-6 (1977) 303 309 (in Japanese)

(5) Ohtani, Y.: "Shadow characteristics under various light source — In the case of a horizontal disk as a shadow caster —_" J. Light \& Vis. Env. 3-1 (1979) 42 51.

(6) Ohtani, Y. and Fujiwara, Y.: "Shadow Characteristics under a Horizontal Disk _ Using a Horizon- tal Square Light Source - $"$ ", J. Illum. Engng. Inst. Japan 64-4 (1980) 183 189 (in Japanese)

(7) Ohtani, Y. and Fujiwara, Y.: "Method of Illuminance Calculation under the Shadow Caster —_ In the Case of a Circline Light Source_- " J. Illum. Engng. Inst. Japan 64-10 (1980) 549 553 (in Japanese)

(8) Ohtani, Y. and Fujiwara, Y.: "Some Characteristics of the Shadow Produced by a Globe as a Shadow Caster Setting under a Horizontal Linear Light Source", J. Illum. Engng. Inst. Japan 70-6 (1986) 249 254 (in Japanese)

(9) Tregenza, P.R.: "The Monte Carlo method in lighting calculation", Light. Res. Technol. 15-4 (1983) 163 170

(10) Stanger, D.: "Monte Carlo procedures in lighting design", J. Illum. Engng. Soc. 13-4 (1984) 368 371

(11) Nagata, M.: "Calculation of Illuminance Distribution in Concave Interior by the Monte Carlo Simulation", J. Illum. Engng. Inst. Japan 72-10 (1988) 596 601 (in Japanese)

(12) Kajiyama, H. and Kodaira, S.: "An illuminance analysis in partitioned spaces using the Monte Carlo Method", J. Illum. Engng. Soc. 18-2 (1989) 93 108

(13) Nagata, M.: "The Calculation Procedure of Illuminance Distribution in an Interior of Finite Width and Infinite Length by the Monte Carlo Simulation", J. Illum. Engng. Inst: Japan 71-6 (1987) 349 354 (in Japanese)

(14) Takubo, A. and Ohtani, Y.: "Illuminance Calculation within the Shadow Affected by Reflected Light", Proc. 1991 Annual Conf. IEI Japan, 104 (in Japanese)

(15) Ohtani, Y., Takubo, A. and Yamaya, T.: "Illuminance Calculation within the Shadow Affected by Reflected Light", J. Illum. Engng. Inst. Japan 76-2 (1992) 96 98 (Research note, in Japanese)

(16) Ohta, K., Ohtani, Y. and Yamaya, T.: "Illuminance Calculation within the Shadow Affected by Reflected Light (part 2)", Proc. 1992 Annual Conf. IEI Japan, 55 (in Japanese)

(17) Ohta, K., Ohtani, Y. and Yamaya, T.: "Illuminance Calculation within the Shadow Affected by Reflected Light (part 3)", Proc. 1992 Tokyo-section Conf. IEI Japan, 23 24 (in Japanese)

(18) Nagata, M.: "Study on Illuminance Distribution and Interreflection in Infinitely Long Concave Interior with Visual Obstructions by Monte Carlo Simulation", Trans, IEE of Japan 109A-12 (1989) 545 552 (in Japanese)

(19) Edited by Illum. Engng. Inst. Japan: "Lighting Handbook", Ohm-sha (1987) 225 (in Japanese)

(20) Norden, K.: "Shadow and Diffusion in Illuminating Engineering", Sir Isaac Pitman \& Sons, Ltd., London (1948) 4

(21) Yamauchi, J. and Nakaji, K.: "Illumination and Electric Heating", Kyouritsu-sha, (1936) 158 (in Japanese)

(Accepted on July 27, 1993) 